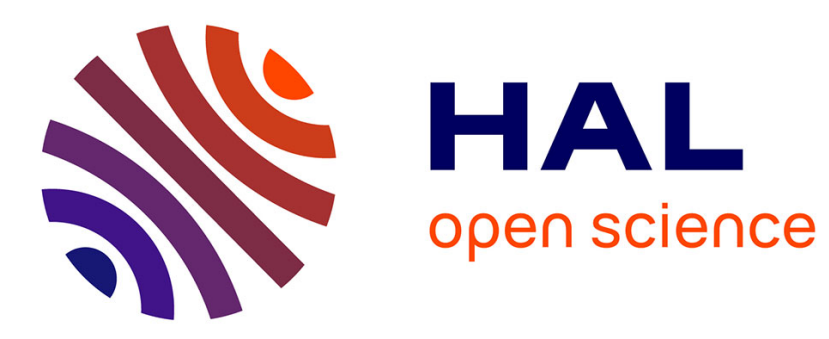

\title{
Microfluidic Channels of adjustable height using deformable elastomer
}

Dandara Valesco Anez, Celine Hadji, Enric Santanach Carreras, Elise Lorenceau, Cyril Picard

\section{- To cite this version:}

Dandara Valesco Anez, Celine Hadji, Enric Santanach Carreras, Elise Lorenceau, Cyril Picard. Microfluidic Channels of adjustable height using deformable elastomer. Microfluidics and Nanofluidics, 2021. hal-03128128

\section{HAL Id: hal-03128128 \\ https://hal.science/hal-03128128}

Submitted on 2 Feb 2021

HAL is a multi-disciplinary open access archive for the deposit and dissemination of scientific research documents, whether they are published or not. The documents may come from teaching and research institutions in France or abroad, or from public or private research centers.
L'archive ouverte pluridisciplinaire HAL, est destinée au dépôt et à la diffusion de documents scientifiques de niveau recherche, publiés ou non, émanant des établissements d'enseignement et de recherche français ou étrangers, des laboratoires publics ou privés. 


\title{
Microfluidic Channels of adjustable height using deformable elastomer
}

\author{
Dandara Velasco Anez • Celine Hadji • \\ Enric Santanach-Carreras • Elise \\ Lorenceau - Cyril Picard
}

Received: date / Accepted: date

\begin{abstract}
In the emerging field of deformable microfluidics, we propose a new geometry in which the height and angle of a channel is controlled thanks to the deformability of the microfluidic elastomer down to thicknesses of a few microns. The particularity of our set-up is that the height of the channel under study is fully closed at rest, which reveals especially well suited to address micro-nanoconfinement problems such as clogging, transport selectivity and flow rectification. Using fluorescence microscopy and light absorption, we probe the channel shape from the measurement of the PDMS-displacement field. We demonstrate that the maximal PDMS-displacement can be inferred from finite elements numerical simulations and predicted reliably with simple analytical relations from elasticity continuum mechanics.
\end{abstract}

Keywords Microfluidics · Fluid-solid interaction · Deformable Microchannel · Shallow microchannel

\section{Introduction}

Soft lithography consists in creating or replicating a master structure using elastomer stamps. This technique makes it possible to manufacture microchan-

Dandara Velasco Anez

Univ. Grenoble Alpes, CNRS, LIPhy, F-38000 Grenoble, France

TOTAL SA, Pôle d'Etudes et Recherche de Lacq, BP 47, 64170, Lacq, France

Enric Santanach-Carreras

Laboratoire Physico-Chimie des Interfaces Complexes, Bâtiment CHEMSTARTUP, Route

Départemental 817, 64170, Lacq, France

TOTAL SA, Pôle d'Etudes et Recherche de Lacq, BP 47, 64170, Lacq, France

Celine Hadji · Elise Lorenceau · Cyril Picard

Univ. Grenoble Alpes, CNRS, LIPhy, F-38000 Grenoble, France

E-mail: cyril.picard@univ-grenoble-alpes.fr 
nels of controlled size and has led to the development of microfluidics. Nowadays, in most researches in this field the elsatomer is poly-dimethylsiloxane (PDMS). PDMS indeed offers several attractive properties such as low price, optical transparency in the visible range, low permeability to polar liquids or electrically insulating properties [1]. The flexibility of PDMS, which allows for an easy release from moulds or conformation to surfaces, has also been proved useful to integrate valves and pumps driven by an external over-pressure $[2,3]$. This paved the way for the emerging domain of deformable microfluidics: by integrating passive elastomeric compliant features, fluidic analog responses of electrical circuits composed of resistors, capacitors, diodes or transistors can now be obtained through the dynamic control of pressure input in PDMS or glass devices $[4,5,6]$. Various complex functions such as micropumps, microflow stabilizers, deformable droplets generators, organ-on-a-chip microfluid models are designed [7]. Deformable microfluidics is also highly interesting when studying particles or cells interactions with solid surfaces. Indeed, modifying channel dimension during the course of an experiment can change the probability of particle deposition on the walls [8] or prevent channel from irreversible clogging as shown in [9], where a deformable membrane microfluidic device allows to clear the clogging of cell aggregates by applying vacuum at the end of the constriction. However, dimensioning these systems correctly, particularly in terms of the pressure required to induce the desired deformation and the pressure gradient required to induce the desired flow, is problematic because the fluid-structure interactions are non-linearly coupled. Therefore, to predict the channel displacement, most of the studies rely on numerical computations based either on a complex non-linear theoretical model, or on a finite element approach $[7,10]$. A compliance scaling parameter $f_{p}$, characterizing the ability of a system to deform has also proven to be useful to relate pressure, and deflection profiles as well as pressure-flow properties [11]. In the reference case of a membrane, the inverse of this parameter is directly proportional to the thickness of the membrane, its Young modulus and the power three of its initial height. Thus, the smaller the Young modulus and the membrane thickness, the higher $f_{p}$ and the membrane deflection. Inspired by these works, we propose a specific geometry for which the height of the channel of interest is vanishingly small when the system is at rest (see Fig. 1b). This has several advantages. First of all, it can be rapidly made since only one lithography step is necessary. Then, $f_{p}$ as defined in [11] is extremely large, thus providing a large sensitivity to pressure variation. Moreover, in this geometry, the height of the channel as well as its angle can be controlled independently by the mean and differential pressure applied apart of the channel. The chosen geometry therefore offers simultaneously a simplicity of realization, control and prediction of the linear and angular displacements by means of simple theoretical expressions. Such a geometry, which may look similar to a microfluidic valve [12], allows to reach almost infinite variation of height, in comparison to previous systems obtained from the deformation of a channel with finite height at rest. Our approach, which aims at controlling precisely the height of a thin channel, distinguishes from the case of a valve, the state of which is either fully 
closed or fully open [12]. The thin channel approach described in this paper reveals especially well suited to address micro-nanoconfinement problems such as clogging, transport selectivity and flow rectification.

Our paper is organized as follows: we first detail the experimental procedure to fabricate and to measure the dimension of the channels. Then using experiments, theory and numerical simulations, we reveal how the amplitude and the angle of the displacement depend on the mean and differential pressure.

\section{Experiments}

As a deformable elastomer, we use Sylgard 184 PDMS (PolyDiMethylSiloxane) which has been cured from a mixture of polymer and crosslinking agent in the proportions 9 to 1 . To retrieve the Young's modulus $E$ of the PDMS elastomer, we measure the deflection of a cantilever made of the same elastomer, clamped on one side and subjected to its own weight on the other, as a function of its length. We obtain the value $E=0.63 \mathrm{MPa}$, in agreement with the values found in the literature for similar proportions of curing agents [13, 14]. The experimental device consists of a PDMS microfluidic chip, formed by two deep adjacent parallel microchannels (pools 1 and 2) with a length $L=1 \mathrm{~cm}$, a width $\ell / 2-w / 2=2.2 \mathrm{~mm}$ and a height $h_{0}=100 \mu \mathrm{m}$ (Fig. 1). These are separated by a PDMS pad of the same length $L$ but with a width $w=60 \mu \mathrm{m}$. The center of the pad is in $x=0, y=0$. Overall, the width of the pad and the two adjacent pools is $\ell=4.5 \mathrm{~mm}$. The height of the whole PDMS device, $H$, obtained from the replication of a SU8-photoresist master structure is 2.5 mm thick.

To seal the PDMS to the glass substrate, most of the glass and PDMS surfaces are exposed to an air-based plasma except for the PDMS pad between the two pools which has been shielded off the plasma. This generates an adhesionfree zone under the pad, while the rest of the channel is safely sealed to the glass slide below. When an over-pressure is applied in the two pools, the elastic PDMS bends and deforms, thus displacing upward this adhesion-free PDMS pad. This opens a thin channel between the two pools as sketched in Fig. 1c). The upper wall of this channel corresponds to the lower surface of the PDMS pad, which is - in the undeformed configuration - in $z=0$, thus in contact with (but not adhering to) the glass slide. Thus, to quantify the PDMS displacement field, we introduce $\mathbf{F}$, which compares the position of a particle located at a position $M(x, y, z)$ in the undeformed configuration and at $M^{\prime}\left(x^{\prime}, y^{\prime}, z^{\prime}\right)$ in the deformed configuration, hence $\mathbf{F}(x, y, z)=\mathbf{M} \mathbf{M}^{\prime}$. Then, we define $F(x, y, z)$ as the projection of $\mathbf{F}(x, y, z)$ along $z$-axis. The lower surfaces of the PDMS are constituted by planes located either at $z=0$ (for the central pad) and $z=h_{0}$ (for the two adjacent pools). Thus, to quantify the displacement field along these PDMS surfaces, we introduce $f$, which writes $f(x, y)=F\left(x, y, z=h_{0}\right)$ for $|y|>w / 2$ and $|x|<L / 2$ and $f(x, y)=F(x, y, z=0)$ for $|y|<w / 2$ and 
$|x|<L / 2$ (note that this last equation also defines the channel height $h$, since the channel only exists for $|y|<w / 2$ and $|x|<L / 2)$.

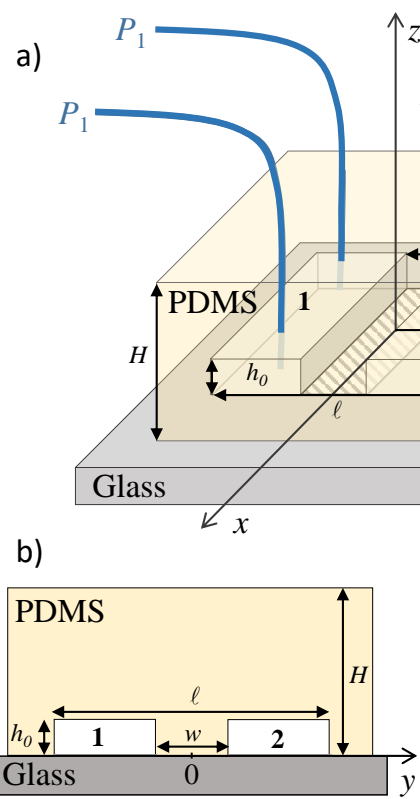

Undeformed configuration

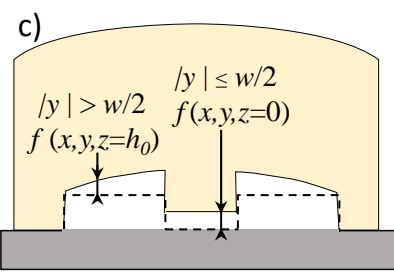

Deformed configuration

Fig. 1 Sketch of the Microfluidic device (not to scale). The typical dimensions of the system are $L=10 \mathrm{~mm}, \ell=4.5 \mathrm{~mm}, w=60 \mu \mathrm{m}, H=2.6 \mathrm{~mm}$ and $h_{0}=100 \mu \mathrm{m}$. a) 3D view of the undeformed configuration. $P_{1}=P_{2}=P_{0}$, the PDMS is not deformed and the hatched $\mathrm{PDMS} /$ glass low adhesion zone is very close from the glass surface. b) Sectional view of the undeformed configuration: $P_{1}=P_{2}=P_{0}$. c) Sectional view of the deformed configuration: $P_{1}>P_{2}>>P_{0}$ the PDMS block is deformed, thus leveling up the hatched zone. This creates a thin channel in between the two pools that can be used to study confined flow.

Each parallel pool has an inlet and an outlet as sketched in Fig. 1. All the inlets and outlets can either be connected to a feeding syringe or a water column through 3 -way valves. The feeding syringes are used to fill up the whole device with liquid, while the water columns are used to impose the pressure in the two pools. We denote as $P_{1}=\rho g H_{1}$ and $P_{2}=\rho g H_{2}$ the over-pressure compared to atmospheric pressure in the pools 1 and 2 , as $\langle P\rangle=\frac{P_{1}+P_{2}}{2}$ the mean over-pressure and as $\Delta P=P_{1}-P_{2}$ the pressure difference between the two pools. With this set-up, a flow driven by pressure difference $\Delta P=P_{1}-P_{2}$ can be induced within a channel of height controlled by $\langle P\rangle$.

To illustrate the correlation between $h$, the channel height in the deformed configuration and $\langle P\rangle$, we set the over-pressure in the two pools at the same positive value after having manually filled up the whole device with syringes. We then wait for 5 minutes, to make sure that the whole system is at equi- 


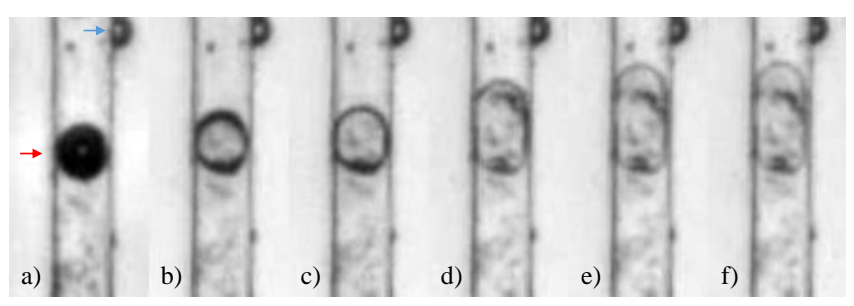

Fig. 2 Images showing the deformation of a bubble stuck under the thin adhesion-free channel when $P_{1}$ and $P_{2}$ are decreased (here $P_{1}=P_{2}$ ). In a) the bubble (highlighted with a red arrow) is dark suggesting a spherical cap. From b) to f), the over-pressure is decreased and the height channel is subsequently diminished. The bubble's shape changes and bridges the two surfaces. Due to contact line pinning, it can only spread in the $x$ direction. The shape of another bubble (highlighted with a blue arrow) sitting on the plane $y=\ell / 2$ remains unchanged. The radius of the bubble highlighted by a red arrow in a) is $30 \mu \mathrm{m}$

librium and that the flow rate under the pad is null. Then, an image of the adhesion-free pad between the two pools is taken with a microscope and the whole procedure is repeated for a lower value of $\langle P\rangle$. In figure 2 , we show the fate of two bubbles when $\langle P\rangle$ is decreased. The first large bubble (seen from above and highlighted with a red arrow) is stuck under the pad while a second smaller one (seen from the edge and highlighted with a blue arrow) is stuck on the vertical wall of the pad. The small bubble appears to be nearly hemispherical whatever $\langle P\rangle$, thus revealing that the contact angle of air in water on the PDMS is nearly $90^{\circ}$. However, the fate of the large bubble is very different. We observe the following features: First, the large bubble in Fig. 2a) is completely dark. This is due to light refraction induced by the index mismatch between air and water and a curved liquid/air interface. Thus, in Fig. 2a), the bubble is a spherical cap. In Fig. 2b), it turns nearly fully transparent but for a dark black edge. This color change is associated to a change in the bubble's geometry. Indeed, in b) the bubble wets the upper and lower surfaces of the channel, thus bridging the two surfaces along an air meniscus, while in a) it is a spherical cap. Since the volume of the small bubble is not modified during the experiment, this change of geometry can only be attributed to a reduction of the channel height associated to the increase of $\langle P\rangle$. From c) to f) the bubble area increases due to a further reduction of the channel height. The spreading only occurs in the $x$ direction: in the $y$ direction, the contact line is presumably pinned on the edge of the limited pad. We also observe a global motion of the bubble along $x$ axis and toward the center of the channel. This step by step motion, induced by the decrease of the channel thickness is not of same nature than the continuous motion of bubbles or drops over surface with a gradient of interfacial energy $[15,16,17,18]$. Indeed, the pinned contact line along $y$ axis as well as high contact angle hysteresis hinder the continuous movement of the bubble [15]. It is only when the bubble is crushed due to the step by step pressure reduction, which perturbs the contact lines, 
that an asymetric movement is observed along the confinement gradient as observed in $[19,20]$. Yet, this demonstrative experiment doesn't allow to retrieve the variation of $f$ (and consequently $h$ for $|y|<w / 2$ and $|x|<L / 2$ ) as a function of $(x, y)$ along the planes $z=0$ and $z=h_{0}$. To do so, we fill up the whole device with water containing Sulforhodamine B at a concentration $2 \mathrm{~g} / \mathrm{L}$. Sulforhodamine B is both a fluorescent molecular dye and a good light absorbent. Contrary to Rhodamine B, which is known to diffuse into PDMS [21], we did not observe any diffusion with Sulforhodamine B. Using these optical properties, we either perform wide-field fluorescence and light absorption measurements. Wide-field fluorescence measurements are performed using an inverted microscope (Olympus - Model IX 11 ), equipped with two different filters. The fluorescence intensity is known to be an increasing function of the number of fluorophore in the region of interest. Using rectangular and square channels of various heights, we compare the measured fluorescence intensity and the thickness of the channels. We note empirically that the intensity of the fluorescence is proportional to the logarithm of the height of the channels with a constant of proportionality such that for channels with a height greater than 15 microns, the measurement uncertainty becomes very large. Indeed in this range, optical aberrations such as out of focus emitted light saturates the signal. In that case, we measure the local thickness of the channel using light absorption and compare the grey-level intensities of transmitted light $I_{t}$ with $h$ the height of calibrated channels filled with Sulforhodamine. These are associated using Beer-Lambert law:

$$
I_{t}=T I_{0} \exp (-\alpha h)
$$

where $\alpha$ has been determined from the light transmission of rectangular tube of controlled height $h(20,30,50,100$ and $200 \mu \mathrm{m})$ filled with the solution, $T$ from the transmission coefficient of a colourless liquid film and $I_{0}$ from the intensity level of the image of the background. We found that the noise to signal ratio is acceptable for channels larger than $10 \mu \mathrm{m}$. These two optical techniques are therefore complementary in our set-up: light absorption measurement being more adapted to "thick" films (larger than $10 \mu \mathrm{m}$ ) and fluorescence measurement to "thin" films (smaller than $15 \mu \mathrm{m}$ ) as in [22]. However, some experimental errors can be expected for the following reasons: first, calibration showed that fluorescence is less reliable for measurements larger than $10 \mu \mathrm{m}$. Second, even though it is not clearly observed, the fluorescence molecules may slightly diffuse in PDMS. Third, refraction occurs due to PDMS deformation. And forth, non homogeneity can be found in the PDMS optical index due to dust or bubbles trapped inside. Therefore, we estimate an uncertainty of $30 \%$ in our experimental measurements.

To complete our understanding of the system, we also perform numerical simulations to quantitatively evaluate $f$ (and consequently $h$ for $|y|<w / 2$ and $|x|<L / 2)$. To do so, we use the 3D Solid model from Structural Mechanics of COMSOL Multiphysics 5.3a in stationary mode using MUMPS (MUltifrontal Massively Parallel sparse direct Solver). PDMS is modeled as a linear elastic (solid isotropic) material, with user-defined properties $(E=0.63 \mathrm{MPa}, \nu=$ 
0.49 and $\left.\rho=970 \mathrm{~kg} / \mathrm{m}^{3}\right)$. The geometrical domain we built, as sketched in Fig. 3, consists on the union of two rectangles (called cantilevers 1 and 2), of same dimension than the PDMS layer above the pools, with a width such that $\ell / 2-w / 2=2.2 \mathrm{~mm}$, a length $L=1 \mathrm{~cm}$ and a thickness $H=2.5 \mathrm{~mm}$. They are linked by a central rectangle (cantilever 3) - representing the pad - $60 \mu \mathrm{m}$ wide, $1 \mathrm{~cm}$ long and $100 \mu \mathrm{m}$ thicker than the other two. The union of these three elements form a whole deformable domain, the four lateral faces of which are fixed and its top surface is set as free. The pressure load, meanwhile, is applied in the two bottom surfaces of the domain. The user-controlled mesh is calibrated for general physics and composed of 298,202 fine-sized elements. The mesh in and around the pad is refined down to $50 \mu \mathrm{m}$ sized elements, with a maximal growth rate of 1.5, reaching the coarse-size elements on the borders of the domain of $200 \mu \mathrm{m}$. Two different types of simulations are run: the first type called "asymmetric" consists on applying a body load of high pressure $\left(P_{1}\right)$ on the bottom face of one of the cantilevers, a low pressure load $\left(P_{2}\right)$ on the bottom face of the other one, and a linear pressure gradient on the bottom face of the pad. Unless specified otherwise, the pressure difference in the asymmetric configuration is fixed and equal to $\Delta P=1620 \mathrm{~Pa}$, while $P_{1}$ varies from $1913 \mathrm{~Pa}$ to $4562 \mathrm{~Pa}$. The second type is the "symmetric" mode and consists on applying the same mean pressure $\langle P\rangle=\left(P_{1}+P_{2}\right) / 2$ on the cantilevers and pad. The simulations are purely statics: the fluid flow dynamics has not been taken into account.

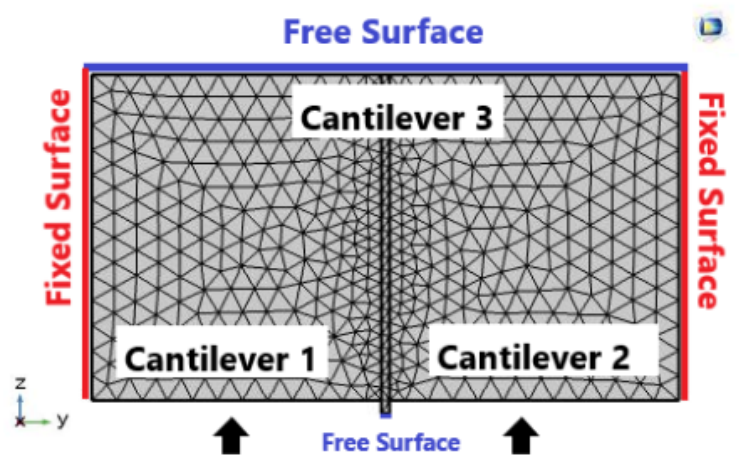

Fig. 3 Cross-sectional view of the geometrical domain created on Comsol. Scheme shows the mesh, as well as the boundary conditions employed on the numerical simulations. Arrows represent the two surfaces where the pressure loads are applied. 


\section{Results}

The surface displacement profiles of the PDMS device are shown in Fig. 4 (a) for $z=h_{0}$. These images are taken for the asymmetric simulation, with $\langle P\rangle=$ $3752 \mathrm{~Pa}$ and $\Delta P=1620 \mathrm{~Pa}$ (hence $P_{1}=4562 \mathrm{~Pa}$ and $P_{2}=2943 \mathrm{~Pa}$ ). The amplitude of the displacement is quantified using the color code on the right of the images and typically ranges between 0 up to $23 \mu \mathrm{m}$. On the bottom face of the PDMS where the pressure load is directly imposed, the color code logically reveals a higher magnitude of surface displacement along pool 1 than on pool 2 since $P_{1}>P_{2}$. However, at the top layer of the PDMS in contact with the atmospheric pressure, the displacement profile is quasi-symmetric. Note that the symmetric simulation, where $P_{1}=P_{2}$, leads to a surface displacement profile which is symmetric on both faces of the PDMS.

We now discuss these observations along $x$ and $y$ axis and compare them with our measurements.

The displacement profile of the bottom surface of the pad, $f$ is measured along $x$-axis for $(y, z)=(0,0)$, a constant $\Delta P=1620 \mathrm{~Pa}$ and $\langle P\rangle$ ranging from $3752 \mathrm{~Pa}$ down to $1104 \mathrm{~Pa}$ (Fig. 5). We use light transmission when the maximal channel height is larger than $15 \mu \mathrm{m}$ and fluorescence when it is smaller than $15 \mu \mathrm{m}$. The displacement of the PDMS along $\mathrm{x}$-axis is logically symmetric and well described by a parabolic shape. This shape is due to the boundary condition : along the edges of the pad, the PDMS is firmly bound to the glass slide, thus preventing the elastomer to deform in this region. Thus, in the numerical simulation, the deformation on the border of the pad, i.e. at $x=-L / 2$ and $x=L / 2$, is null. However, experimental results show a minimal displacement different from zero in $x=-L / 2$. This is due to optical aberrations caused by refraction of the fluorescence in the PDMS as can be seen on the top of Fig.5, where an image of the channel taken in fluorescence microscopy reveals heterogeneities in the light intensity. From these curves, we introduce the maximal PDMS-displacement, $f_{m}=f(x=0, y=0, z=0)$. In Fig.5, $f_{m}$ ranges from $6 \mu \mathrm{m}$ for $\langle P\rangle=1104 \mathrm{~Pa}$ up to $20 \mu \mathrm{m}$ for $\langle P\rangle=3752$ Pa.

The displacement profile of the bottom surface of the pad, $f$, is presented along $y$-axis for $x=0$ and $z=h_{0}$ in Fig. 4(b) for asymmetric simulations with $\Delta P=1620 \mathrm{~Pa}$ and symmetric simulations with $\Delta P=0 \mathrm{~Pa}$ and $\langle P\rangle$ between $1104 \mathrm{~Pa}$ and $3752 \mathrm{~Pa}$. Quite surprisingly, we overall observe that the displacement field in the vicinity of the pad is identical in the two simulation configurations, which is presumably due to the symmetry of the boundary condition. We also observe that the dashed red line showing the displacement profile along the top surface $(z=H)$ is more symmetric than the continuous red line showing the displacement along the bottom surface $\left(z=h_{0}\right)$. Indeed the asymmetry of the deformation, which generates internal stresses within the PDMS, has become more uniform thanks to the great thickness of the elastomer layer.

To check that the channel displacement only depends on $\langle P\rangle$ and is therefore decoupled from the flow driven by $\Delta P$, the displacement profiles along $y$ 


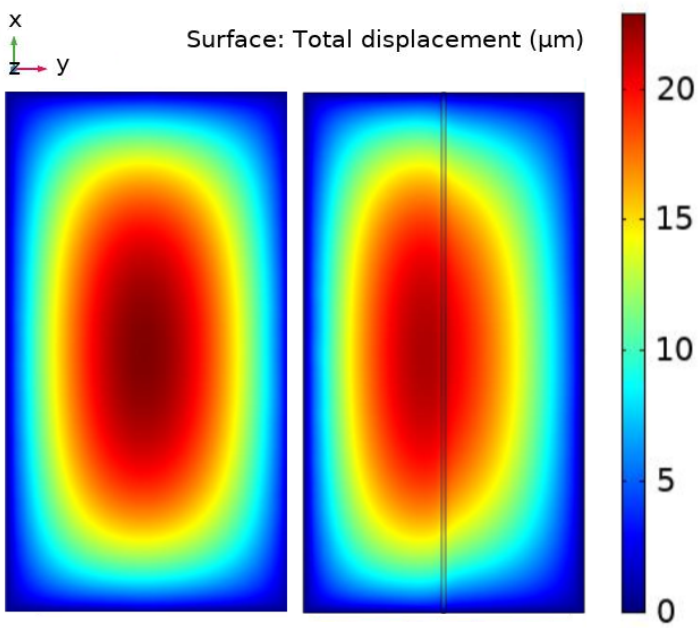

(a)

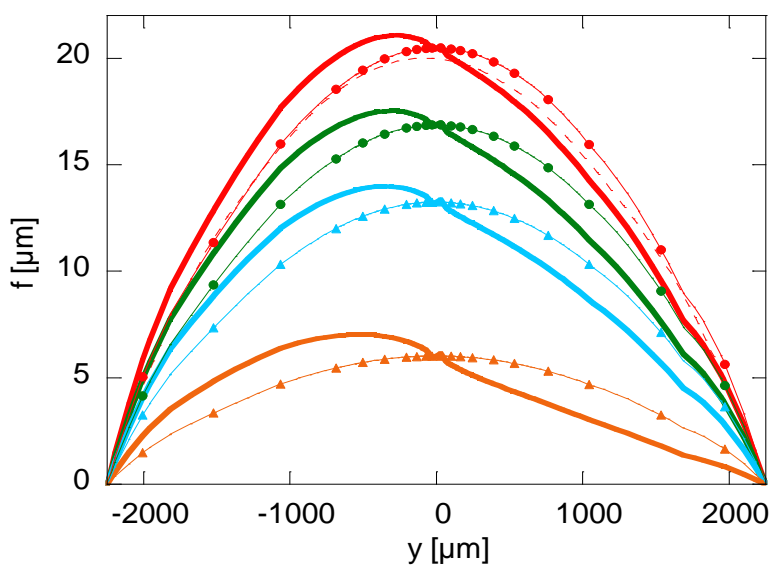

(b)

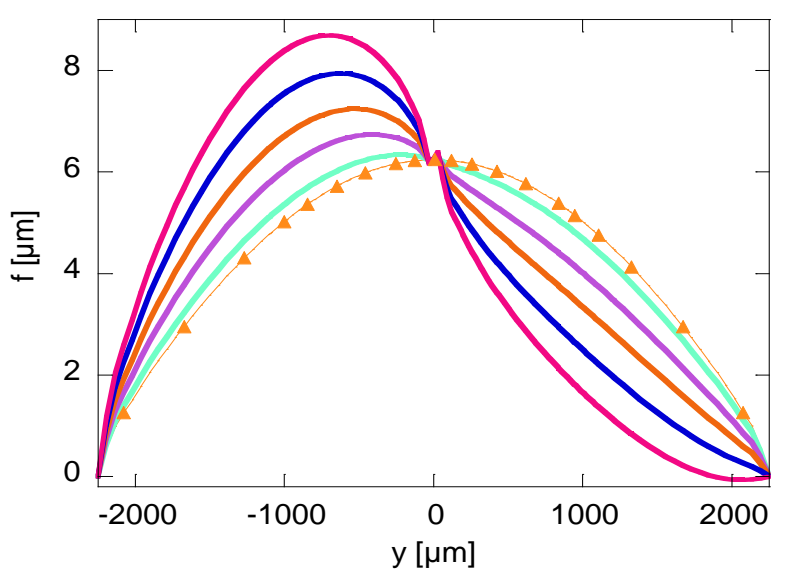

(c)

Fig. 4 Comsol simulation of the deformation of the system. (a) Total surface displacement profiles on top $(z=H)$ and bottom surfaces $\left(z=h_{0}\right)$, respectively, for $\Delta P=1620 \mathrm{~Pa}$ and $\langle P\rangle=3752 \mathrm{~Pa}$. The position of the pad is highlighted on the right by two lines. (b) Line field displacement profiles $f$ along $y$-axis for $x=0$ and various values of $\langle P\rangle$ and $z$. Red: $\langle P\rangle=3752 \mathrm{~Pa}$, Green: $\langle P\rangle=3090 \mathrm{~Pa}$, Blue: $\langle P\rangle=2428 \mathrm{~Pa}$, Orange: $\langle P\rangle=1104 \mathrm{~Pa}-$ Dotted lines: $\Delta P=0 \mathrm{~Pa}$ and $z=h_{0}$, Full lines: $\Delta P=1620 \mathrm{~Pa}$ and $z=h_{0}$. Dashed line: $\Delta P=1620 \mathrm{~Pa}$ and $z=H$ (c) Line field displacement profiles $f$ along $y$-axis for $x=0$, $z=h_{0}$ and $\langle P\rangle=1104 \mathrm{~Pa}$. Dotted Orange line: $\Delta P=0 \mathrm{~Pa}$, Magenta: $\Delta P=3237 \mathrm{~Pa}$, Blue: $\Delta P=2428 \mathrm{~Pa}$, Full orange line: $\Delta P=1620 \mathrm{~Pa}$, Purple: $\Delta P=971 \mathrm{~Pa}$, Light Green: $\Delta P=324 \mathrm{~Pa}$. 


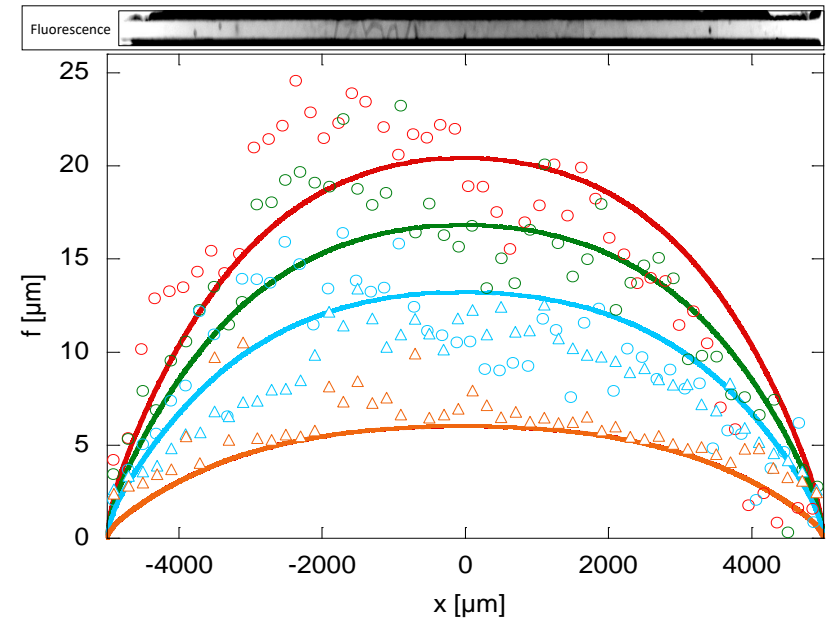

Fig. 5 Experimental and simulated line field displacement profiles along $x$-axis for $y=0$, $z=0$ (i.e. at the center of the channel) and $\Delta P=1620 \mathrm{~Pa}$. Red: $\langle P\rangle=3752 \mathrm{~Pa}$, Green: $\langle P\rangle=3090 \mathrm{~Pa}$, Blue: $\langle P\rangle=2428 \mathrm{~Pa}$, Orange: $\langle P\rangle=1104 \mathrm{~Pa}$. Dots: light transmission measurements, Triangles: fluorescence measurements, Lines: Comsol simulations.

for $\Delta P$ varying between 0 and $3237 \mathrm{~Pa}$ while maintaining a constant $\langle P\rangle=$ $1104 \mathrm{~Pa}$, is presented in Fig. 4(c). At this rather large scale, we observe that all the data indeed collapse at the center of the channel, thus demonstrating that it is only $\langle P\rangle$ that controlled the channel height. Moreover, the greater $\Delta P$, the greater the asymmetry between pool 1 and pool 2 .

At the scale of the channel, the experimental and numerical displacements measured and computed for a given $\Delta P$ are not perfectly perpendicular to the $x$ axis as can be seen in Fig. 6(a). This means that the channel is slightly inclined. We define $f_{m}$ as the mean value of $f$ for $x=0$ and $|y|<w / 2$. The difference between $f$ and $f_{m}$, which indicates the local amplitude of the tilt is normalized by $f_{m}$ and plotted in Fig. 6(b) as a function of $y$ for different $\Delta P$ while maintaining $\langle P\rangle$ constant.

To go one step further, we compare the value of the displacement field $f_{m}$ obtained in simulations and experiments in Fig. 7(a). We observe an excellent quantitative agreement between the two. This demonstrates that the mechanical model used in the simulations to describe the experimental set-up is accurate. Moreover, modeling the deformable PDMS elastomer above the channels as a unique cantilever of volume $\left(H-h_{0}\right) L \ell$ supported in $y= \pm \frac{\ell}{2}$ and on which a uniform pressure $\langle P\rangle$ is applied, we compute the maximal deformation of this system $f_{m}$ from classical analytical results from linear elasticity:

$$
f_{m}=\frac{5\langle P\rangle \ell^{4}}{32 E\left(H-h_{0}\right)^{3}}
$$




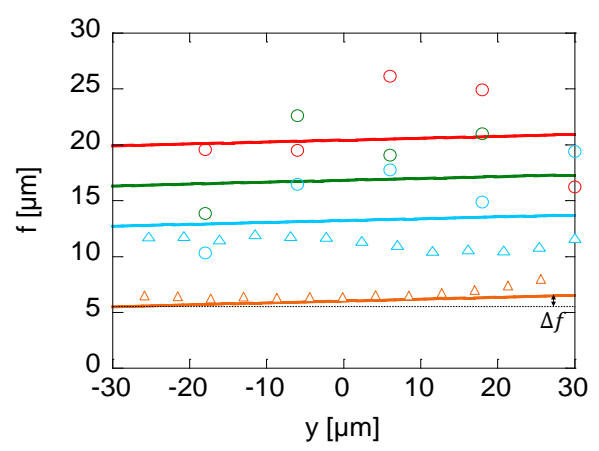

(a)

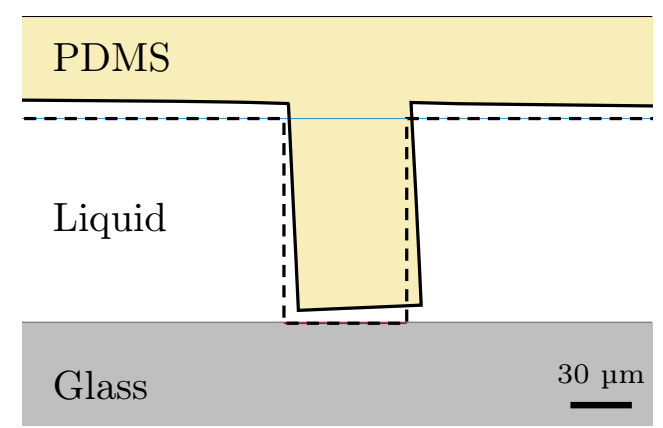

(c)

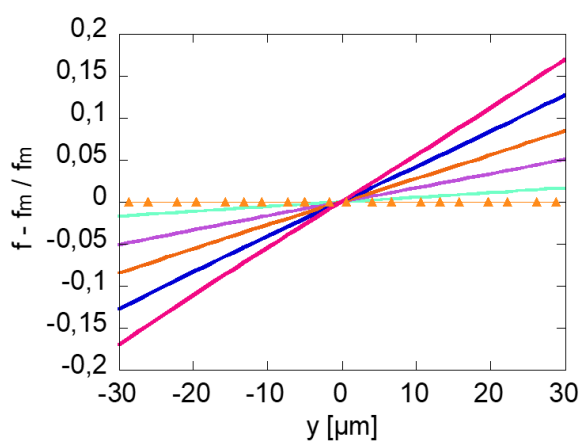

(b)

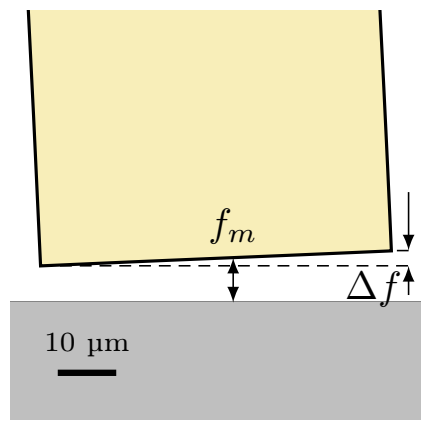

(d)

Fig. 6 (a) Experimental and simulated line field displacement profiles $f$ along the y-axis for $x=0, z=0$ and $|y|<w / 2$. Color coding of data corresponds to Fig. 5. From this data, we define $f_{m}$ as the average value of $f$ for $|y|<w / 2$ and $x=0$ (b) Difference between $f$ and $f_{m}$ normalized by $f_{m}$ along $y$-axis for $x=0, z=0$ and $|y|<w / 2$. Extracted by numerical simulations. Color coding of data corresponds to Fig. 4(c). (c) Reconstruction of the shape of the PDMS at the vicinity of the membrane obtained from Comsol simulation. Dashed line: $\langle P\rangle=0 \mathrm{~Pa}$ and $\Delta P=0 \mathrm{~Pa}$ (undeformed configuration). Full line: $\langle P\rangle=1104 \mathrm{~Pa}$ and $\Delta P=3237 \mathrm{~Pa}$ (Magenta case in figures 4(c) and 6(b)). Graphs in figure 4 correspond to displacements of the upper plane of the pools $\left(z=h_{0}\right.$ : blue line) while graphs in the figure $6(\mathrm{a})$ and 6 (b) correspond to displacement of the base of the membrane $(z=0$ : red line). (d) Zoom of the channel formed by the displacement of the membrane, the average height of which is $f_{m}$ and the difference in height between inlet and outlet is $\Delta f$.

where $E$ is the Young Modulus of the material and $H-h_{0}$ is the cantilever's thickness. Due to the boundary conditions chosen in this model, one notes that the cantilever behaves as an uni-dimensional system and the Poisson ratio of the material does not play any role.

Analytic results from Eq.2, experimental measurements and numerical simulations, all shown in Fig. 7(a), are in fair agreement even though the analytic calculation doesn't take into account the pressure difference $\Delta P$ along the channel nor the presence of the extra $h_{0}$-thick pad in $(x, z)=(0,0)$ and $|y|<h / 2$. Yet, analytical results are slightly larger than Comsol and experimental results, probably because deformations along the $x$-axis are neglected 
in the model due to the small $\ell / L$ aspect ratio. In other words, the model does not take into account clamping in the $x$-direction which slightly limits the deformation of the channel at its center.

Nevertheless, using this framework, it is not only possible to retrieve analytically $f_{m}$, but also the amplitude of the tilt observed in the membrane as $\Delta P$ increases. To do so, we compute the displacement field induced when applying a pressure load $\Delta P$ along a smaller cantilever of volume $h_{0} L w$ clamped in $z=h_{0}$ as sketched in Fig.6(c). We introduce $\Delta f$ as the difference of deformation magnitude along the width $w$ of the membrane, given by:

$$
\Delta f=\frac{2 \Delta P h_{0}^{3}}{E w^{2}}
$$

Combining Eq.2 and 3, we obtain an expression that relates directly the deformation to the pressure ratio $\Delta P /\langle P\rangle$ to $N$, with $N=h_{0}^{3}\left(H-h_{0}\right)^{3} /\left(l^{4} w^{2}\right)$ being a dimensionless number characteristic of the geometry of the microfluidic device.

$$
\frac{\Delta f}{f_{m}}=\frac{64}{5} \frac{\Delta P}{\langle P\rangle} \frac{h_{0}^{3}\left(H-h_{0}\right)^{3}}{l^{4} w^{2}}=\frac{64}{5} \frac{\Delta P}{\langle P\rangle} N
$$

$\Delta f / f_{m}$ is plotted as a function of $\Delta P /\langle P\rangle$ in Fig. 7(b). Here again, we observe a good agreement between the analytical expression of Eq.4 and the simulations since in both cases, there is a linearity between $\Delta P /\langle P\rangle$ and $\Delta f / f_{m}$. Moreover, they quantitatively agree within less than $15 \%$. This remarkable property makes it possible to analytically predict the height of the deformed channel, and thus to overcome complex numerical simulations. This good agreement also suggests that the deformation of the system is strongly related to its dimensions which can be adjusted to favor or not the asymmetry of the channel according to the value of the dimensionless number $N$. For small values of $N$ the height of the channel is mainly fixed by the average pressure and not the difference of pressure across the pad, this geometrical parameter can be fully fixed independently of the flow and its evolution in time. It is thus, easy to decouple the control of the pad of the system from the characterization of flow and transport phenomena. For large value of $N$, the asymmetry of the channel is expected to become significant even for moderate difference of pressure.

However, the values of the observed slopes are slightly different: the slope of the analytical calculation $64 / 5 \times N$ systematically overestimate that of the numerical simulations, closer to $64 / 5.85 \times N$. This observation is surprising: Figure 7 (a) shows that for a given value of $\langle P\rangle$, the analytically calculated $f_{m}$ is always higher than the simulated $f_{m}$ due to boundary conditions across the cantilever not accounted for in the analytical calculation. However, here, although $\Delta f$ is normalized by $f_{m}$, the same trend is still observed: the result of the analytical calculation is always larger than the numerical calculation. This suggests that the $\Delta f$ calculated by Eq. 4 overestimates by far the $\Delta f$ simulated by Comsol, probably due to the curvature of the cantilever along the $x$-axis not taken into account in the analytical calculation. 


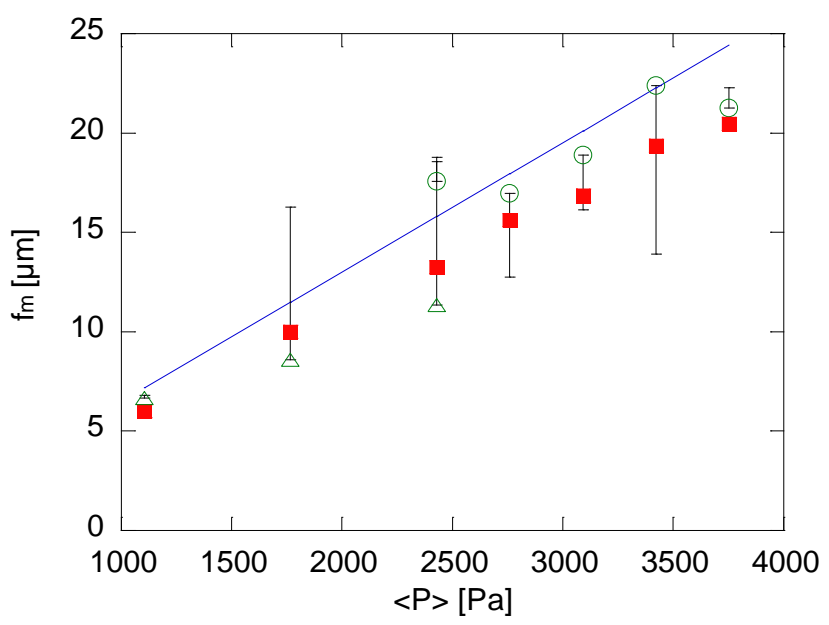

(a)

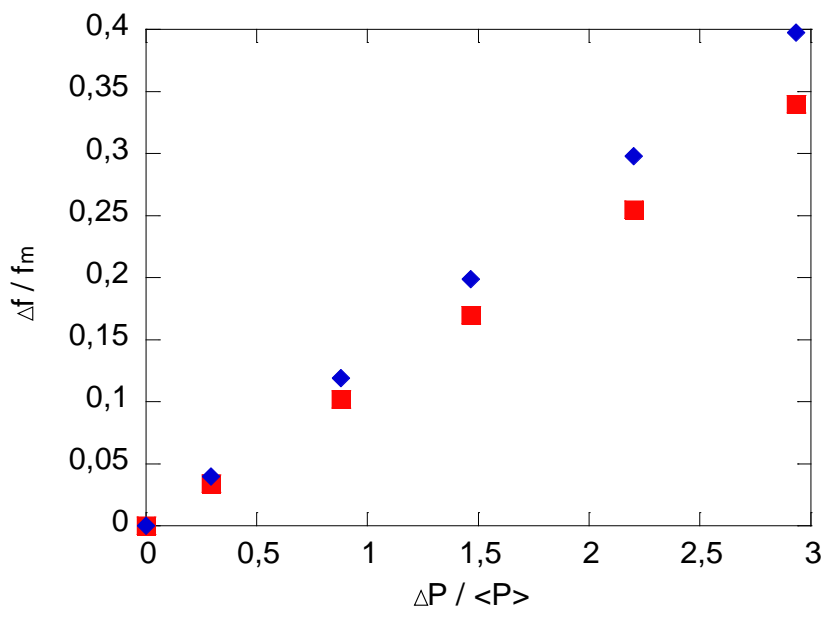

(b)

Fig. 7 (a) $f_{m}$ as a function of $\langle P\rangle$ obtained from experiments with $\Delta P=1620 \mathrm{~Pa}$ (open green symbols: circles for light absorption measurements and triangles for fluorescence measurements), Comsol simulations with $\Delta P=1620 \mathrm{~Pa}$ (red squares) and analytical calculations with $\Delta P=0 \mathrm{~Pa}$ (blue line). (b) Difference of deformation over maximal deformation, in function of difference of pressure over average over-pressure, for Comsol simulations with $\Delta P=1620 \mathrm{~Pa}$ (red squares) and analytical calculations with $\Delta P=0 \mathrm{~Pa}$ (blue rhombi).

\section{Conclusion}

We show that the proposed study geometry makes it possible to obtain channels whose height is i) low - typically of the order of a few microns although achieved by soft photolithography techniques ii) modulated by the average pressure of the flow whatever the pressure difference along it iii) variable or 
not according to the value of the dimensionless number $N$. It is thus, easy to decouple the control of channel height from the characterization of flow and transport phenomena. Moreover, our approach gives the ability to finely tune this asymmetry and study its impact upon above mentioned surface-induced effects.

The obtained results here pave the way for the usage of micro- nano-fluidic veins with tunable size of interest for the characterization of the flow of complex fluids such as polymer solutions or colloidal suspensions. We demonstrate that the height of a PDMS microchanel can be finely adjusted in a predictive manner with a simple pressure control down to almost micron size. The system gives the ability to study, in a reversible manner, various effects related to adsorption/adhesion on the wall of the channel. For instance, one may study the impact of depletion and change in fluid concentration, the enhancement of slippage or clogging events. In standard microfluidic systems, clogging is generally irreversible, but it might not be the case in a deformable system. Transient complex processes, can be finely studied, back and forth, according to the channel height. It is thus possible to characterize nonlinear hysteric phenomena such as clogging and unclogging. Beyond pressure-driven flow, electro-osmotic phenomena could be also considered. In particular the asymmetric configuration could be of interest to study rectification effects. To go beyond the PDMS case, Eq. 2 shows that the height is inversely dependent on the material's Young modulus. Using much rigid material such as silicon, may offer the possibility to control a nanogap size of interest for the study of nanofluidic phenomena.

We thus show, on the considered range of pressure, that the channel height changes by a factor of three starting at a value of $6 \mu \mathrm{m}$. This value demonstrates the ability of the system to reach strong confinement even if based on soft lithography.

Acknowledgements We sincerely thank Jean-Claude Vial, Aurelien Gourrier and Irene Wang for their time and help obtaining successful quantitative optical measurements. We also appreciate Saranath Seshadri's support on Comsol utilisation and Daniele Centanni for microfluidic preparation. We acknowledge Elisabeth Charlaix and Benjamin Cross for stimulating discussions and TOTAL SA for funding.

\section{Conflict of interest}

The authors declare that they have no conflict of interest.

\section{References}

1. Sindy K .Y. Tang and George M. Whitesides. Basic microfluidic and soft lithographic techniques. McGraw-Hill, New-York, 7 edition, 2100. 
2. Marc A Unger, Hou-Pu Chou, Todd Thorsen, Axel Scherer, and Stephen R Quake. Monolithic microfabricated valves and pumps by multilayer soft lithography. Science, 288(5463):113-116, 2000.

3. Todd Thorsen, Sebastian J Maerkl, and Stephen R Quake. Microfluidic large-scale integration. Science, 298(5593):580-584, 2002.

4. Daniel C. Leslie, Christopher J. Easley, Erkin Seker, James M. Karlinsey, Marcel Utz, Matthew R. Begley, and James P. Landers. Frequency-specific flow control in microfluidic circuits with passive elastomeric features. $\mathrm{Na}$ ture Physics, 5(3):231-235, 2009.

5. W Grover, A Skelley, C Liu, E Lagally, and R Mathies. Monolithic membrane valves and diaphragm pumps for practical large-scale integration into glass microfluidic devices. Sensors and Actuators, B: Chemical, 89: 315-323, 2003.

6. J Weaver, J Melin, D Stark, S Quake, and M Horowitz. Static control logic for microfluidic devices using pressure-gain valves. Nature Physics, 6(3):218-223, 2010.

7. A. Raj, Pearlson P. A. Suthanthiraraj, and A. K. Sen. Pressure-driven flow through pdms-based flexible microchannels and their applications in microfluidics. Microfluidics and Nanofluidics, 22(11):128, 2018. doi: 10.1007/s10404-018-2150-5.

8. Cesare M. Cejas, Fabrice Monti, Marine Truchet, Jean-Pierre Burnouf, and Patrick Tabeling. Universal diagram for the kinetics of particle deposition in microchannels. Phys. Rev. E, 98:062606, Dec 2018. doi: 10.1103/PhysRevE.98.062606. URL https://link.aps.org/doi/10.1103/PhysRevE.98.062606.

9. Song-Bin Huang, Yang Zhao, Deyong Chen, Hsin-Chieh Lee, Yana Luo, Tzu-Keng Chiu, Junbo Wang, Jian Chen, and Min-Hsien Wu. A clogging-free microfluidic platform with an incorporated pneumatically driven membrane-based active valve enabling specific membrane capacitance and cytoplasm conductivity characterization of single cells. Sensors and Actuators: B. Chemical, 190(Complete):928-936, 2014. doi: 10.1016/j.snb.2013.09.070.

10. A. Mehboudi and J. Yeom. Experimental and theoretical investigation of a low-reynolds-number flow through deformable shallow microchannels with ultra-low height-to-width aspect ratios. Microfluidics and Nanofluidics, 23 (5), 2019.

11. A. Raj and A. K. Sen. Flow-induced deformation of compliant microchannels and its effect on pressure-flow characteristics. Microfluidics and Nanofluidics, 20(2), FEB 2016. doi: 10.1007/s10404-016-1702-9.

12. Guru Prasath Natarajan, Sung-Jin Kim, and Chang-Wan Kim. Analysis of membrane behavior of a normally closed microvalve using a fluid-structure interaction model. Micromachines, 8(355):1-15, 2017.

13. Joong Park, Sung Yoo, Eun-Joong Lee, Dae Ho Lee, Ji Young Kim, and Sang-Hoon Lee. Increased poly(dimethylsiloxane) stiffness improves viability and morphology of mouse fibroblast cells. BioChip Journal, 4, 09 2010. doi: $10.1007 / \mathrm{s} 13206-010-4311-9$. 
14. Deniz K. Armani, C. Liu, and Narayana R Aluru. Re-configurable fluid circuits by pdms elastomer micromachining. Technical Digest. IEEE International MEMS 99 Conference. Twelfth IEEE International Conference on Micro Electro Mechanical Systems (Cat. No.99CH36291), pages 222227, 1999.

15. MK Chaudhury and GM Whitesides. How to make water run uphill. Science, 256(5063):1539-1541, JUN 121992.

16. E Lorenceau and D Quere. Drops on a conical wire. Journal of Fluid Mechanics, 510:29-45, 2004.

17. Etienne Reyssat. Drops and bubbles in wedges. Journal of Fluid Mechanics, 748:641-662, 2014.

18. Yongmei Zheng, Hao Bai, Zhongbing Huang, Xuelin Tian, Fu-Qiang Nie, Yong Zhao, Jin Zhai, and Lei Jiang. Directional water collection on wetted spider silk. NATURE, 463(7281):640-643, FEB 42010.

19. Ashutosh Shastry, Marianne J. Case, and Karl F. Bohringer. Directing droplets using microstructured surfaces. Langmuir, 22(14):6161-6167, JUL 42006.

20. P. Brunet, J. Eggers, and R. D. Deegan. Vibration-induced climbing of drops. Physical Review Letters, 99(14), 2007.

21. JL Pittman, CS Henry, and SD Gilman. Experimental studies of electroosmotic flow dynamics in microfabricated devices during current monitoring experiments. Anal. Chem., 75(3):361-370, 2003.

22. P. Petit, J. Seiwert, I. Cantat, and A.-L. Biance. On the generation of a foam film during a topological rearrangement. Journal of Fluid Mechanics, 763:286-301, JAN 2015.

23. Sindy KY Tang and George M Whitesides. Basic microfluidic and soft lithographic techniques, 2010.

24. Bobak Mosadegh, Tom Bersano, Joong Park, Mark A Burns, and Shuichi Takayama. Next-generation integrated microfluidic circuits. Lab on a chip, 11:2813-8, 092011.

25. M. Reyssat, F. Pardo, and D. Quere. Drops onto gradients of texture. EPL, 87(3), 2009. 